\title{
Review of: "Neuroaffective profiles are associated with e- cigarette use"
}

\author{
Laura Miccoli
}

Potential competing interests: Dr Versace and I coauthored some scientific works.

The use of k-means cluster analysis on neural reactions to addiction and affective cues has emerged as a theoretically and methodologically powerful tool to identify substance users based on the motivational relevance each of them assigns to diverse stimuli, related or not to addiction. The manuscript by Versace and Kypriotakis confirms the existence of these neural profiles, further providing behavioral data that support their prognostic significance.

The theoretical relevance of this technique lies not only in identifying a subgroup of substance users characterized by hyper-reactivity to addiction cues, but also in showing their simultaneous decrease of motivation during intrinsically pleasant stimuli (i.e., erotic pictures). From a clinical standpoint, this anomalous neural profile indicates that for those individuals addiction-related cues have become more important than some of the most universally positive contents. This pattern of reactions to drug-related and pleasant cues has been observed in individuals addicted to, e.g., cocaine, tobacco, and food; the pattern is absent in the other subgroup of 'healthier' addicts, showing the typical greatest neural reactivity to erotica and lower responsivity to drug-related cues. Strikingly, the 'healthier' subgroup has a better prognosis and a higher probability of quitting.

Although limited by the consequences of the Covid-19 pandemic, that prevented the authors from gathering data from a larger sample, Versace and Kypriotakis' manuscript is a valuable contribution to this line of research. The ms is clear, brief and to the point, pleasant to read. The data emerged confirm the solidity of the profiles while also reporting, most notably, that they correspond to different behaviors during a 'nicotine availability task': Addicts whose neural profile shows exaggerated reactions to tobacco cues and reduced reactions to erotica take more nicotine puffs than addicts with the standard neural profiles. Therefore, the neural profiles distinguish the most severe from the 'healthier' addicts and result in different behaviors, as a whole substantiating the scientific feasibility and clinical relevance of tailored treatments.

I annotate here a couple of marginal doubts/suggestions.

- In the Results, the authors write: "The 2 groups did not differ according to demographic characteristics, nicotine dependence, mood, or impulsivity". I am wondering whether this implies that there were differences based on some of the remaining features.

- Still in the Results, "For all 3 estimated quantiles, the $\mathrm{C}>\mathrm{P}$ group took a significantly higher number of puffs than the $P>C$ group. Specifically, the differences in the estimated number of puffs 
were $5(\mathrm{C}>\mathrm{P}=9$ vs. $\mathrm{P}>\mathrm{C}=4), 5(\mathrm{C}>\mathrm{P}=15$ vs. $\mathrm{P}>\mathrm{C}=10)$, , and $8(\mathrm{C}>\mathrm{P}=35$ vs. $\mathrm{P}>\mathrm{C}=27)$, puffs at the 25th, 50th, and 75th percentiles, respectively."

Given the importance of the finding, I am wondering whether the authors could increase the clarity of the paragraph (maybe by shortening it and including the second part -from "Specifically" on- in the first?).

Also, there is a typo (,,).

- Looking at Figure 1, it seems that the C>P group showed or tended to show, compared to the $\mathrm{P}>\mathrm{C}$ group, also lower LPP to UL/Unpleasant cues Low in motivation. I am interested in whether the authors could assess this tendency in their previous studies. In case such tendency was indeed confirmed in the current and/or previous studies but the authors would regard it as clinically not meaningful, I would appreciate if they could provide a rationale.

- There is a mismatch in the pic acronyms: IAPS food pics are reported as food/FD in 'Materials (cued nicotine selfadministr task)' and Figure 1 but as PS in Supplem. Table 2.

Since these are pleasant cues that correspond to UO/Unpleasant Objects, they might be labeled as PF/Pleasant Foods or any other label that might suit the authors and be consistently used throughout the ms.

- It was a bit confusing that the authors used slightly different labels for at least partially overlapping aspects of the task: "cued nicotine availability task", "electronic nicotine delivery system/ENDS", "cued nicotine self-administration task", "ENDS cues", "EC/electronic cigarette cues".

I am wondering whether the authors might increase the consistency of the labels and, if possible, choose only one or two of the most straightforward labels (e.g., "nicotine availability cues" or "EC cues/electronic cigarette cues"; up to the authors' discretion). 